\title{
OPEN Multi-symplectic integrator of the generalized KdV-type equation based on the variational principle
}

\author{
Yi Wei $^{1 *}$, Xing-Qiu Zhang ${ }^{1}$, Zhu-Yan Shao ${ }^{1}$, Jian-Qiang Gao ${ }^{1}$ \& Xiao-Feng Yang ${ }^{2}$ \\ The variational principle is used to construct a multi-symplectic structure of the generalized KdV-type \\ equation. Accordingly, the local energy conservation law, the local momentum conservation law, and \\ the Cartan form of the generalized KdV-type equation are given. An explicit multi-symplectic scheme \\ for the generalized KdV equation based on the Fourier pseudo-spectral method and the symplectic \\ Euler scheme is constructed. Through a numerical examination, the explicit multi-symplectic Fourier \\ pseudo-spectral scheme for the generalized KdV equation not only preserve the discrete global energy \\ conservation law and the global momentum conservation law with high accuracy, but show long-time \\ numerical stability as well.
}

In this paper, we aim to study the generalized KdV-type equation in the form

$$
u_{t}+f(u) u_{x}+\left(g\left(u_{x}\right)\right)_{x x}=0,
$$

where $f$ and $g$ are smooth functions.

When $f(u)=\alpha u^{\lambda}$ and $g\left(u_{x}\right)=\delta u_{x}$, Eq. (1.1) reduces to the generalized KdV equation

$$
u_{t}+\alpha u^{\lambda} u_{x}+\delta u_{x x x}=0,
$$

where $\alpha, \delta$, and $\lambda$ are arbitrary constants.

Setting $\lambda=1$, Eq. (1.2) reduces to the KdV equation ${ }^{1,2}$

$$
u_{t}+\alpha u u_{x}+\delta u_{x x x}=0 .
$$

Setting $\lambda=2$, Eq. (1.2) reduces to the mKdV equation ${ }^{1,2}$

$$
u_{t}+\alpha u^{2} u_{x}+\delta u_{x x x}=0 .
$$

The KdV equation is originally used to describe long waves propagating in a channel. The KdV equation can also describes the propagation of plasma waves in a dispersive medium ${ }^{2,3}$. The $\mathrm{KdV}$ equation and the $\mathrm{mKdV}$ equation are most popular soliton equations which have been extensively studied ${ }^{2,4-11}$, because these two equations possess many interesting properties of mechanism and geometry ${ }^{12}$. Based on the homogeneous balance of undetermined coefficients method (HBUCM $)^{13,14}$, Yang et al. ${ }^{13}$ proposed the definition and a multi-symplectic structure of the generalized KdV-type equation. Obviously, the KdV equation, the mKdV equation and the generalized $\mathrm{KdV}$ equation are special cases of the generalized $\mathrm{KdV}$-type equation.

To understand the mechanism of complex physical phenomena, we should obtain solutions of the generalized $\mathrm{KdV}$-type equation. There are some methods to obtain exact solutions, such as the first integral method ${ }^{15}$, the $\left(G^{\prime} / G\right)$-expansion $\operatorname{method}^{9}$, the homogeneous balance method ${ }^{16}$, the modified simple equation $\operatorname{method}^{17}$, simplified Hirota's method ${ }^{18}$, and so on. However, the generalized KdV-type equation is a nonlinear partial differential equation (NLPDE), it is difficult to obtain the exact solutions or there is no exact solution. In these cases, it is natural to resort to the numerical methods ${ }^{13}$. A large amount of numerical methods have been applied on the $\mathrm{KdV}$ equation and the $\mathrm{mKdV}$ equation in the last few years, including quintic B-Spline basis functions ${ }^{19}$, finite difference scheme ${ }^{20}$, symplectic method ${ }^{12}$, multi-symplectic Preissmann box scheme ${ }^{6}$, multi-symplectic box schemes ${ }^{21}$, and so on.

\footnotetext{
${ }^{1}$ School of Medical Information Engineering, Jining Medical University, Rizhao, 276826, Shandong, P.R. China. ${ }^{2}$ College of Science, Northwest A\&F University, Yangling, 712100, Shaanxi, P. R. China. *email: weiyiwy@126.com
} 
Many physical properties of a system are closely related to the geometric structure of the equation ${ }^{22}$. This naturally requires that numerical methods can preserve exactly geometric structure during the simulation. Multi-symplectic method possesses the stability and effectiveness, and can preserve the multi-symplectic structure of the Hamiltonian system ${ }^{14,23}$. In the present paper, a multi-symplectic structure of the generalized KdV-type equation is given by the variational principle. To obtain a multi-symplectic structure of the generalized KdV-type equation based on variational principle, we need to cast Eq. (1.1) into a system of equations. Therefore, it will be helpful to give a detailed derivation of a multi-symplectic structure for the generalized KdV-type equation since this derivation will provide some guiding principles in finding multi-symplectic structures for other NLPDEs.

We also consider a multi-symplectic Fourier pseudo-spectral discretization for Eq. (1.2) and demonstrate its convergence by simulating the evolution of the soliton. The remainder of this paper is organized as follows. In section 2, a multi-symplectic structure of the generalized KdV-type equation is derived based on the variational principle. An explicit multi-symplectic Fourier pseudo-spectral scheme for the generalized KdV equation is given in section 3. In section 4, the solitary wave behaviors of the generalized KdV equation are simulated. In section 5, some conclusions are given.

\section{Multi-Symplectic Structure of The Generalized Kdv-Type Equation Based on The Variational Principle}

In this section, a multi-symplectic structure of the generalized KdV-type equation is given by the variational principle. The covariant configuration space for Eq. (1.1) is denoted by $X \times U$, where $X=(x, t)$ represents the space of independent variables and $U=(\phi, u, v, \omega, \sigma)$ represents the space of dependent variables. The internal variables $\phi, u, v, \omega$, and $\sigma$ are defined to construct a multi-symplectic structure of the generalized KdV-type equation. The first order prolongation of $X \times U$ is defined to be $U^{(1)}=X \times U \times U_{1}$, where $U_{1}=\left(\phi, u, v, \omega, \sigma, \phi_{x}, u_{x}, v_{x}\right.$, $\left.\omega_{x}, \sigma_{x}, \phi_{t}, u_{t}, v_{t}, \omega_{t}, \sigma_{t}\right)$ represents the space consisting of the first order partial derivatives. Let $\varsigma: X \rightarrow U$ be a smooth function and we suppose $\varsigma \in H^{2}[X] \times H^{2}[X]$, where $H^{2}[X]$ is the second order Sobolev space defined on $X$. Then, its first prolongation is denoted by $\operatorname{pr}^{1} \varsigma=\left(\phi, u, v, \omega, \sigma, \phi_{x}, u_{x}, v_{x}, \omega_{x}, \sigma_{x}, \phi_{t}, u_{t}, v_{t}, \omega_{t}, \sigma_{t}\right)$. The Lagrangian density for Eq. (1.1) is

$$
L\left(\operatorname{pr}^{1}(\varsigma)\right)=L\left(\operatorname{pr}^{1}(\varsigma)\right) d x \wedge d t
$$

where

$$
L\left(\operatorname{pr}^{1}(\varsigma)\right)=-2 \iint f(u) d^{2} u-u \omega-u \phi_{t}+2 \sigma u_{x}+\phi_{x} \omega+2 \int g(v) d v-2 \sigma v .
$$

Corresponding to the Lagrangian density (2.2), the action functional is defined by

$$
S(\varsigma)=\int_{M} L\left(\operatorname{pr}^{1}(\varsigma)\right)
$$

where $\varsigma \in H^{2}[M] \times H^{2}[M]$ and $M$ is an open set in $X$.

Let $V$ be a vector field on $X \times U$ with the form

$$
\begin{aligned}
V= & \xi(x, t) \frac{\partial}{\partial t}+\eta(x, t) \frac{\partial}{\partial x}+\tau(x, t, \phi, u, v, \omega, \sigma) \frac{\partial}{\partial \phi}+\varphi(x, t, \phi, u, v, \omega, \sigma) \frac{\partial}{\partial u} \\
& +\vartheta(x, t, \phi, u, v, \omega, \sigma) \frac{\partial}{\partial v}+\rho(x, t, \phi, u, v, \omega, \sigma) \frac{\partial}{\partial \omega}+\gamma(x, t, \phi, u, v, \omega, \sigma) \frac{\partial}{\partial \sigma} .
\end{aligned}
$$

The flow $\exp (\beta V)$ of the vector field $V$ is a one-parameter transformation group of $X \times U$. The map $\varsigma: M \rightarrow U$ and a family of maps $\widetilde{\varsigma}: \widetilde{M} \rightarrow U$ depend on the parameter $\beta$. Now, the variation of the action functional (2.1) is calculated as follows:

$$
\begin{aligned}
\delta S= & \left.\frac{d}{d \beta}\right|_{\beta=0} S(\widetilde{\varsigma}) \\
= & \left.\frac{d}{d \beta}\right|_{\beta=0} \int_{\widetilde{M}} L\left(\operatorname{pr}^{1}(\widetilde{\varsigma})\right) \\
= & \left.\frac{d}{d \beta}\right|_{\beta=0} \int_{\widetilde{M}}\left(-2 \iint f(\widetilde{u}) d^{2} \widetilde{u}-\widetilde{u} \widetilde{\omega}-\widetilde{u} \widetilde{\phi}_{\tilde{t}}+2 \widetilde{u_{\tilde{u}}}\right. \\
& \left.+\widetilde{\phi}_{\widetilde{x}} \widetilde{\omega}+2 \int g(\widetilde{v}) d \widetilde{v}-2 \widetilde{\sigma} \widetilde{v}\right) d \widetilde{x} \wedge d \tilde{t} \\
= & \int_{M} A d x \wedge d t+B,
\end{aligned}
$$

where

$$
\begin{aligned}
A= & \xi\left(E_{t}+F_{x}\right)+\eta\left(I_{t}+G_{x}\right)+\tau\left(u_{t}-\omega_{x}\right)+\varphi\left(-\phi_{t}-2 \sigma_{x}-2 \int f(u) d u-\omega\right) \\
& +\vartheta(2 g(v)-2 \sigma)+\rho\left(\phi_{x}-u\right)+\gamma\left(2 u_{x}-2 v\right),
\end{aligned}
$$




$$
\begin{gathered}
B=\int_{\partial M}(\xi(E d x-F d t)+\eta(I d x-G d t)+\tau(\omega d t+u d x)+\varphi(2 \sigma d t)), \\
E=2 \iint f(u) d^{2} u-2 \int g(v) d v, F=\omega \phi_{t}+2 \sigma u_{t}, I=-u^{2}, \\
G=2 \iint f(u) d^{2} u+u \omega+u \phi_{t}-2 \int g(v) d v+2 \sigma v .
\end{gathered}
$$

If $\xi, \eta, \tau, \phi, \vartheta, \rho$, and $\gamma$ have compact support on $M$, then $B=0$. In this case, with the requirement of $\delta S=0$ and from Eq. (2.5), the variation $\xi$ yields the local energy conservation law

$$
E_{t}+F_{x}=0
$$

and the variation $\eta$ yields the local momentum conservation law

$$
I_{t}+G_{x}=0
$$

where $E, F, I$, and $G$ are same as to Eq. (2.8).

For a conservative $L$, i.e., one that does not depend on $x$ and $t$ explicitly, Eqs (2.9) and (2.10) become the local energy conservation law and the local momentum conservation law, respectively ${ }^{5}$.

The variations $\tau, \phi, \vartheta, \rho$, and $\gamma$ yield the Euler-Lagrange equation

$$
\begin{gathered}
u_{t}-\omega_{x}=0, \\
-\phi_{t}-2 \sigma_{x}=2 \int f(u) d u+\omega, \\
0=2 \sigma-2 g(v), \\
\phi_{x}=u, \\
2 u_{x}=2 v .
\end{gathered}
$$

If the condition that $\xi, \eta, \tau, \sigma, \theta, \vartheta$, and $\varsigma$ having compact support on $M$ is not imposed, then from the boundary integral $B$, the Cartan form can be defined as

$$
\begin{aligned}
\Theta_{L}= & (\omega d \phi+2 \sigma d u) \wedge d t+u d \phi \wedge d x \\
& +\left(2 \int g(v) d v-2 \iint f(u) d^{2} u-u \omega-2 \sigma v\right) d x \wedge d t
\end{aligned}
$$

which satisfies (denote the interior product and pull back mapping as $\rfloor$ and ()$\left.^{*}\right)$

$$
B=\int_{\partial M}\left(\operatorname{pr}^{1} \varsigma\right)^{*}\left\langle\operatorname{pr}^{1} V \downarrow \Theta_{L}\right\rangle .
$$

The multi-symplectic form of the generalized KdV-type equation is defined to be

$$
\Theta_{L}=d \Theta_{L}
$$

Remark 1 Equation (2.11) is equivalent to a multi-symplectic structure of generalized KdV-type Eq. (1.1) as follows:

$$
\boldsymbol{M}_{5} z_{t}+\boldsymbol{K}_{5} z_{x}=\nabla_{z} S(z)
$$

where

$$
\boldsymbol{M}_{5}=\left[\begin{array}{ccccc}
0 & 1 & 0 & 0 & 0 \\
-1 & 0 & 0 & 0 & 0 \\
0 & 0 & 0 & 0 & 0 \\
0 & 0 & 0 & 0 & 0 \\
0 & 0 & 0 & 0 & 0
\end{array}\right], \boldsymbol{K}_{5}=\left[\begin{array}{ccccc}
0 & 0 & 0 & -1 & 0 \\
0 & 0 & 0 & 0 & -2 \\
0 & 0 & 0 & 0 & 0 \\
1 & 0 & 0 & 0 & 0 \\
0 & 2 & 0 & 0 & 0
\end{array}\right], z=\left[\begin{array}{c}
\phi \\
u \\
v \\
\omega \\
\sigma
\end{array}\right]
$$

and Hamiltonian function $S(z)=u \omega+2 \sigma v-2 \int g(v) d v+2 \iint f(u) d^{2} u$.

Remark 2. The multi-symplectic structure (2.15) is identical to the results by using the HBUCM ${ }^{13}$. Moreover, the Cartan form of the generalized KdV-type equation can be obtained by the variational principle.

Remark 3. Obviously, a multi-symplectic structure of the generalized KdV Eq. (1.2) is 


$$
\begin{aligned}
u_{t}-\omega_{x} & =0 \\
-\phi_{t}-2 \delta v_{x} & =\frac{2 \alpha u^{\lambda+1}}{(\lambda+1)}+\omega, \\
2 \delta u_{x} & =2 \delta v 0 \\
\phi_{x} & =u
\end{aligned}
$$

or

$$
\boldsymbol{M}_{4} z_{t}+\boldsymbol{K}_{4} z_{x}=\nabla_{z} S(z)
$$

where

$$
\boldsymbol{M}_{4}=\left[\begin{array}{cccc}
0 & 1 & 0 & 0 \\
-1 & 0 & 0 & 0 \\
0 & 0 & 0 & 0 \\
0 & 0 & 0 & 0
\end{array}\right], \boldsymbol{K}_{4}=\left[\begin{array}{cccc}
0 & 0 & 0 & -1 \\
0 & 0 & -2 \delta & 0 \\
0 & 2 \delta & 0 & 0 \\
1 & 0 & 0 & 0
\end{array}\right], \boldsymbol{z}=\left[\begin{array}{c}
\phi \\
u \\
v \\
\omega
\end{array}\right]
$$

and Hamiltonian function $S(\boldsymbol{z})=\frac{2 \alpha u^{\lambda+2}}{\left(\lambda^{2}+3 \lambda+2\right)}+\delta v^{2}+u \omega$.

According to the multi-symplectic theory presented by Bridges ${ }^{24-27}$, the multi-symplectic conservation law in the wedge product form, the local energy conservation law and the local momentum conservation law for the generalized KdV Eq. (1.2) are as follows:

$$
\theta_{t}+\kappa_{x}=0, E_{t}+F_{x}=0, G_{x}+I_{t}=0,
$$

where

$$
\begin{gathered}
\theta=d \phi \wedge d u, \kappa=-d \phi \wedge d \omega-2 \delta d u \wedge d v \\
E=\frac{2 \alpha u^{\lambda+1}}{\lambda^{2}+3 \lambda+2}-\delta v^{2}, F=\phi_{t} \omega+2 \delta v u_{t}, G=\frac{2 \alpha u^{\lambda+2}}{\left(\lambda^{2}+3 \lambda+2\right)}+\delta v^{2}+u \omega+u \phi_{t}, I=-u^{2} .
\end{gathered}
$$

\section{Explicit Multi-Symplectic Fourier Pseudo-Spectral Method For The Generalized Kdv Equation}

In order to derive the algorithms conveniently, we introduce some notations: $x_{i}=x_{L}+i h, t_{j}=j \tau(i=0,1, \ldots, N-1$; $j=0,1, \ldots)$, where $h=\frac{x_{R}-x_{L}}{N}=\frac{L}{N}$ and $\tau$ are spatial and temporal step lengths, the indexes $i$ and $j$ denote the discrete space and time dimensions. Denote $u_{i}^{j}$ as the approximate value of $u\left(x_{i}, t_{j}\right)$. As we know, the first-order differential operator $\partial_{x}$ yields the Fourier spectral differentiation matrix $\boldsymbol{D}$. Here, $\boldsymbol{D}$ is an $N \times N$ anti-symmetry matrix with elements ( $N$ is an even number)

$$
(\boldsymbol{D})_{r, s}=\left\{\begin{array}{lr}
\frac{1}{2} \mu(-1)^{r+s} \cot \left(\mu \frac{x_{r}-x_{s}}{2}\right), & r \neq s, \\
0, & r=s,
\end{array}\right.
$$

where $r=1,2, \ldots, N$ and $s=1,2, \ldots, N$ represent column and row of the matrix $\boldsymbol{D}$, and $\mu=\frac{2 \pi}{L}$. For more details, one can consult refs. ${ }^{11,28}$ and references therein.

Using the notations

$$
\boldsymbol{\Phi}=\left[\begin{array}{c}
\phi_{0} \\
\phi_{1} \\
\vdots \\
\phi_{N-1}
\end{array}\right], \boldsymbol{u}=\left[\begin{array}{c}
u_{0} \\
u_{1} \\
\vdots \\
u_{N-1}
\end{array}\right], \boldsymbol{v}=\left[\begin{array}{c}
v_{0} \\
v_{1} \\
\vdots \\
v_{N-1}
\end{array}\right], \boldsymbol{\omega}=\left[\begin{array}{c}
\omega_{0} \\
\omega_{1} \\
\vdots \\
\omega_{N-1}
\end{array}\right], \boldsymbol{u}^{\lambda+1}=\left[\begin{array}{c}
\left(u_{0}\right)^{\lambda} \\
\left(u_{1}\right)^{\lambda} \\
\vdots \\
\left(u_{N-1}\right)^{\lambda}
\end{array}\right]
$$

and discretizing the multi-symplectic structure of the generalized KdV Eq. (2.17) with the Fourier pseudo-spectral method in the space domain, the discrete form of the generalized KdV Eq. (1.2) can be obtained as follows:

$$
\begin{gathered}
\frac{d \boldsymbol{u}}{d t}-D \boldsymbol{\omega}=0, \\
-\frac{d \boldsymbol{\Phi}}{d t}-2 \delta \boldsymbol{D} \boldsymbol{v}=\frac{2 \alpha \boldsymbol{u}^{\lambda+1}}{\lambda+1}+\boldsymbol{\omega}, \\
2 \delta \boldsymbol{D} \boldsymbol{u}=2 \delta \boldsymbol{v}, \\
\boldsymbol{D} \boldsymbol{\Phi}=\boldsymbol{u} .
\end{gathered}
$$

Theorem 1. The Fourier pseudo-spectral semi-discretization (3.3) has $N$ semi-discrete multi-symplectic conservation laws 


$$
\frac{d \chi_{i}}{d t}+\sum_{k=0}^{N-1}(D)_{i, k} \boldsymbol{\zeta}_{i, k}=0,(i=1,2, \cdots, N),
$$

where $\chi_{i}=\frac{1}{2} d z_{i} \wedge \boldsymbol{M} d z_{i}, \zeta_{i}=\frac{1}{2} d z_{i} \wedge \boldsymbol{K} d z_{i}, z_{i}=\left[u_{i}, \varphi_{i}, \omega_{i}, \rho_{i}, v_{i}\right]^{\mathrm{T}}$, index $i$ represent $i$ th equation and is from 1 to $N$.

Proof. Equation (3.3) can be re-written as a compact form

$$
\boldsymbol{M} \frac{d \boldsymbol{z}_{i}}{d t}+\boldsymbol{K} \sum_{k=0}^{N-1}(\boldsymbol{D})_{i, k} \mathbf{z}_{k}=\nabla_{\mathbf{z}} S\left(\boldsymbol{z}_{i}\right) .
$$

The variational equation associated with Eq. (3.5) is

$$
\boldsymbol{M} \frac{d}{d t} d \mathbf{z}_{i}+\boldsymbol{K} \sum_{k=0}^{N-1}(\boldsymbol{D})_{i, k} d \boldsymbol{z}_{k}=S_{z z}\left(z_{i}\right) d z_{i} .
$$

Taking the wedge product with $d z_{i}$ on both sides of Eq. (3.6) and noticing

$$
d z_{i} \wedge S_{z z}\left(z_{i}\right) d z_{i}=0
$$

thus, we show the $N$ semi-discrete multi-symplectic conservation laws.

Because $\boldsymbol{D}$ is anti-symmetry and $\boldsymbol{\zeta}_{i, k}=\zeta_{k, i}$, summing Eq. (3.6) over the spatial index yields

$$
\frac{d}{d t} \sum_{j=0}^{N-1} \chi_{j}=0
$$

which implies conservation of the total symplecticity over time. Thus, it is natural to integrate with respect to time by using a symplectic integrator ${ }^{13,29}$.

Discretizing Eq. (3.3) with respect to the time domain by the symplectic Euler scheme yields

$$
\boldsymbol{M}_{+} \delta_{t}^{+} \boldsymbol{z}_{i}^{j}+\boldsymbol{M}_{-} \delta_{t}^{-} \boldsymbol{z}_{i}^{j}+\boldsymbol{K} \sum_{k=0}^{N-1}(\boldsymbol{D})_{i, k} \boldsymbol{z}_{k}^{j}=\nabla_{z} S\left(\boldsymbol{z}_{i}^{j}\right),
$$

where $\delta_{t}^{+}$and $\delta_{t}^{-}$are the forward and backward difference operators, respectively:

$$
\delta_{t}^{+} \boldsymbol{z}_{i}^{j}=\frac{\boldsymbol{z}_{i}^{j+1}-\boldsymbol{z}_{i}^{j}}{\tau}, \delta_{t}^{-} \boldsymbol{z}_{i}^{j}=\frac{\boldsymbol{z}_{i}^{j}-\boldsymbol{z}_{i}^{j-1}}{\tau},
$$

and $\boldsymbol{M}_{+}$and $\boldsymbol{M}_{-}$are the matrices splitting for the symplectic structure matrix $\boldsymbol{M}$,

$$
\boldsymbol{M}=\boldsymbol{M}_{+}+\boldsymbol{M}_{-}, \quad \boldsymbol{M}_{+}^{\mathrm{T}}=-\boldsymbol{M}_{-} .
$$

The matrix splitting of $\boldsymbol{M}$ is not unique ${ }^{13,29}$. Here, taking $\boldsymbol{M}_{+}$as an upper triangle matrix

$$
\boldsymbol{M}_{+}=-\boldsymbol{M}_{-}^{\mathrm{T}}=\left[\begin{array}{llll}
0 & 1 & 0 & 0 \\
0 & 0 & 0 & 0 \\
0 & 0 & 0 & 0 \\
0 & 0 & 0 & 0
\end{array}\right],
$$

an efficient stable explicit scheme for the generalized KdV Eq. (1.2) is obtained as follows:

$$
\begin{gathered}
\left(\frac{u_{i}^{j+1}-u_{i}^{j}}{\tau}\right)-\boldsymbol{D}\left(\boldsymbol{\omega}_{k}^{j}\right)_{i}=0, \\
-\left(\frac{\phi_{i}^{j}-\phi_{i}^{j-1}}{\tau}\right)-2 \delta \boldsymbol{D}\left(\boldsymbol{v}_{k}^{j}\right)_{i}=\frac{2 \alpha\left(u_{i}^{j}\right)^{\lambda+1}}{\lambda+1}+\omega_{i}^{j}, \\
2 \delta\left(\boldsymbol{D} \boldsymbol{u}_{k}^{j}\right)_{i}=2 \delta v_{i}^{j}, \\
\left(\boldsymbol{D} \Phi_{k}^{j}\right)_{i}=u_{i}^{j} .
\end{gathered}
$$

Eliminating the auxiliary variables and re-writing the equations, a compact form is obtained as follows:

$$
\frac{1}{2}\left(\frac{u_{i}^{j+1}-u_{i}^{j-1}}{\tau}\right)+\delta\left(\boldsymbol{D}^{3} u_{k}^{j}\right)_{i}+\alpha\left(u^{\lambda}\right)_{k}^{j}\left(\boldsymbol{D} u_{k}^{j}\right)_{i}=0, \quad(i=1, \cdots, N-1) .
$$

where $i$ is spatial index and $\boldsymbol{D}^{3}=\boldsymbol{D} \cdot \boldsymbol{D} \cdot \boldsymbol{D}$. 
Theorem 2. The discrete scheme (3.13) has $N$ full-discrete multi-symplectic conservation laws

$$
\frac{\chi_{i}^{j}-\chi_{i}^{j-1}}{\tau}+\sum_{k=0}^{N-1}(D)_{i, k} \zeta_{k}^{j}=0,(i=1,2, \cdots, N),
$$

where $\boldsymbol{\chi}_{i}^{j}=\frac{1}{2} d \boldsymbol{z}_{i}^{j} \wedge \boldsymbol{M}_{+} d \boldsymbol{z}_{i}^{j+1}, \boldsymbol{\zeta}_{k}^{j}=\frac{1}{2} d \boldsymbol{z}_{i}^{j} \wedge \boldsymbol{K} d \boldsymbol{z}_{k}^{j}$, index $i$ representation $i$ th equation.

Proof From theorem 1 and Eqs (3.6) and (3.13) can be re-written as a compact form

$$
\boldsymbol{M}_{+} \frac{\boldsymbol{z}_{i}^{j+1}-\boldsymbol{z}_{i}^{j}}{\tau}+\boldsymbol{M}_{-} \frac{\boldsymbol{z}_{i}^{j}-\boldsymbol{z}_{i}^{j-1}}{\tau}+\boldsymbol{K} \sum_{k=0}^{N-1}(\boldsymbol{D})_{i, k} \boldsymbol{z}_{k}^{j}=\nabla_{\mathbf{z}} S\left(\boldsymbol{z}_{i}^{j}\right) .
$$

The variational equation associated with Eq. (3.16) is

$$
\boldsymbol{M}_{+} \frac{d \boldsymbol{z}_{i}^{j+1}-d \boldsymbol{z}_{i}^{j}}{\tau}+\boldsymbol{M}_{-} \frac{d \boldsymbol{z}_{i}^{j}-d \boldsymbol{z}_{i}^{j-1}}{\tau}+\boldsymbol{K} \sum_{k=0}^{N-1}(\boldsymbol{D})_{i, k} d \boldsymbol{z}_{k}^{j}=S_{z z}\left(\boldsymbol{z}_{i}^{j}\right) d \boldsymbol{z}_{i}^{j} .
$$

Taking the wedge product with $d z_{i}^{j}$ on both sides of Eq. (3.17) and noticing

$$
d z_{i}^{j} \wedge S_{z z}\left(z_{i}^{j}\right) d z_{i}^{j}=0 .
$$

then, the $N$ full-discrete multi-symplectic conservation laws are verified ${ }^{13,29}$.

\section{Numerical Experiment}

In this section, we conduct a typical numerical experiment for the scheme (3.14) to verify the theoretical conclusions, including the accuracy, the ability to preserve the local energy conservation law and the local momentum conservation law of the generalized KdV Eq. (1.2) for long-time integration.

Applying the Riccati-Bernoulli sub-ODE method ${ }^{30}$ to the generalized KdV Eq. (1.2), has an exact solution as follows:

$$
u=\left(\frac{A \delta\left(\lambda^{2}+3 \lambda+2\right)}{2 \alpha \lambda} \operatorname{sech}^{2}\left(\frac{\sqrt{A}}{2} \xi\right)\right)^{\frac{1}{\lambda}}, \xi=x-\frac{A \delta}{\lambda^{2}} t
$$

where $A$ is an arbitrary constant.

Inserting the parameters $\lambda=\sqrt{2}, \alpha=1, \delta=1$ and $A=2$ into Eq. (1.2), we obtain

$$
u_{t}+u^{\sqrt{2}} u_{x}+u_{x x x}=0 \text {, }
$$

which has the exact traveling wave solution ( $\operatorname{set} \lambda=\sqrt{2}, \alpha=1, \delta=1$ and $A=2$ in Eq. (3.19))

$$
u(x, t)=\left(\frac{(4+3 \sqrt{2})}{2} \operatorname{Sech}^{2}\left(\frac{\sqrt{2}}{2}(x-t)\right)\right)^{\frac{1}{\sqrt{2}}} .
$$

The space interval is $\left[x_{L}, x_{R}\right]=[-20,40]$ with the periodic boundary condition

$$
u(x-20, t)=u(x+40, t),
$$

and the initial condition

$$
u(x, 0)=\left(\frac{(4+3 \sqrt{2})}{2} \operatorname{Sech}^{2}\left(\frac{\sqrt{2}}{2} x\right)\right)^{\frac{1}{\sqrt{2}}} .
$$

We fix the space step $h=0.2$ and the time step $\tau=1 \times 10^{-4}$ for the scheme (3.14). Based on the multi-symplectic theory of Bridges ${ }^{24-27}$, the global energy $E(t)$ and the global momentum $I(t)$ of the generalized KdV Eq. (3.20) with the periodic boundary condition (3.22) and the initial condition (3.23) are written as

$$
E(t)=\int_{-20}^{40}\left(\frac{2 u^{\sqrt{2}+2}}{4+3 \sqrt{2}}-u_{x}^{2}\right) d x, I(t)=-\int_{-20}^{40} u^{2} d x .
$$

Accordingly, $E^{j}$ and $I^{j}$, which denote the discrete global energy and the global momentum on the $j$ th time level, are written as 

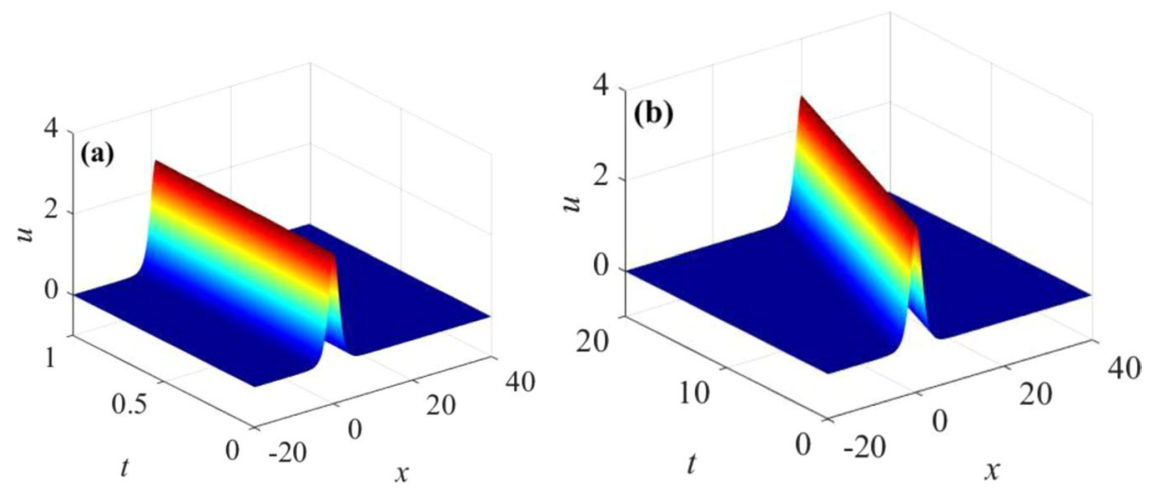

Figure 1. Three-dimensional waveform of numerical solution: (a) the numerical solution with time $0 \leq t \leq 1$, (b) the numerical solution with time $0 \leq t \leq 20$.
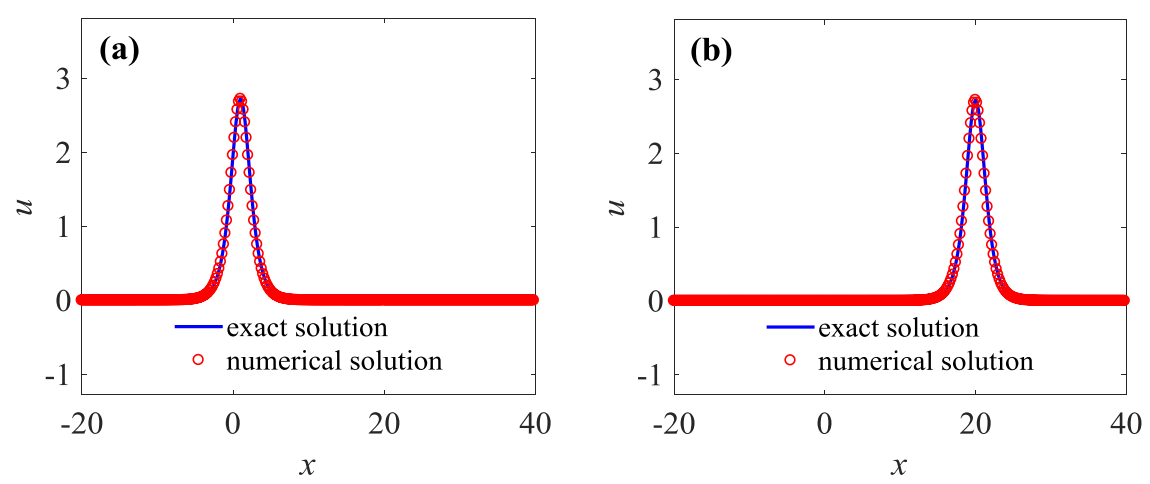

Figure 2. Two-dimensional waveform of numerical solution and exact solution: (a) The exact solution and the numerical solution with time $t=1,(\mathbf{b})$ The exact solutions and the numerical solutions with time $t=20$.

$$
\begin{aligned}
& E^{j}=\frac{1}{5} \sum_{i=1}^{N}\left(\frac{2}{4+3 \sqrt{2}}\left(\frac{u_{i}^{j}+u_{i-1}^{j}}{2}\right)^{\sqrt{2}+2}-\left(\frac{\left(\boldsymbol{D} \boldsymbol{u}_{k}^{j}\right)_{i}+\left(\boldsymbol{D} \boldsymbol{u}_{k}^{j}\right)_{i-1}}{2}\right)^{2}\right), \\
& I^{j}=-\frac{1}{5} \sum_{i=1}^{N}\left(\frac{u_{i}^{j}+u_{i-1}^{j}}{2}\right)^{2} .
\end{aligned}
$$

The errors of the discrete global energy conservation law Error $E$ and the global momentum conservation law Error $I$ on the $j$ th time level are defined as follows:

$$
\text { Error } E=E^{j}-E^{0}, \text { Error } I=I^{j}-I^{0},
$$

where $E^{0}$ and $I^{0}$ are the initial value of the discrete global energy and the global momentum.

Now we define the error as follows:

$$
\text { Error }=\max _{0 \leq i \leq N-1}\left\|u\left(x_{i}, j \tau\right)-u_{i}^{j}\right\|_{\infty} .
$$

Applying the scheme (3.14) to simulate the generalized KdV Eq. (3.20) with the periodic boundary condition (3.22) and the initial conditions (3.23) up to $t=20$, three-dimensional waveform figure of numerical solutions (Fig. 1a $(t=1)$ and Fig. $1 \mathrm{~b}(t=20))$, two-dimensional waveform figure of numerical solutions (Fig. 2a $(t=1)$ and Fig. $2 \mathrm{~b}(t=20)$ ), three-dimensional error figure (Fig. 3a), two-dimensional error figure (Fig. 3b), the global energy error figure (Fig. 4a) and the global momentum error figure (Fig. 4b) are obtained as follows:

Figure 1 shows that waveform of numerical solutions does not change with time by applying the multi-symplectic Fourier pseudo-spectral method to Eq. (3.20). It indicates that the basic geometric properties of Eq. (3.20) can be well maintained by the numerical soliton solutions. From Fig. 2a,b, $u$ decreases gradually and tends to zero with $x \rightarrow \infty$, which is consistent with the exact solution (3.21), and the numerical solutions nearly overlap the exact solution, which implies high accuracy of the multi-symplectic Fourier pseudo-spectral scheme. From the Fig. 3a,b, it is shown that the errors between the exact solution and the numerical solution are up to $10^{-9}$, and the errors are mainly from the boundary conditions. From Fig. 4a,b, the multi-symplectic Fourier pseudo-spectral method to the generalized $\mathrm{KdV}$ equation can well maintain two important geometric properties 

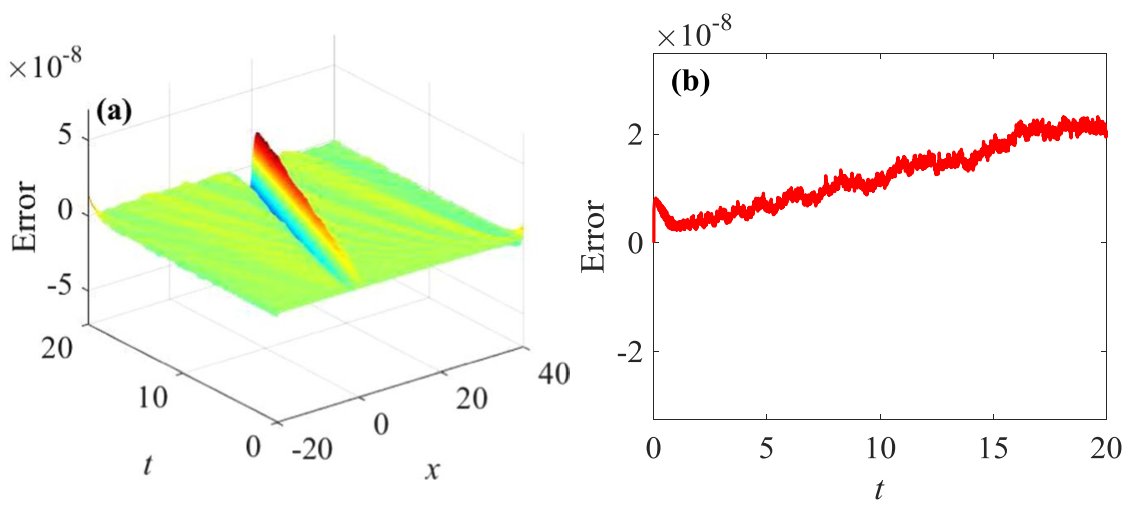

Figure 3. Errors with time $0 \leq t \leq 20$ : (a) three-dimensional errors with time, (b) two-dimensional errors with time.
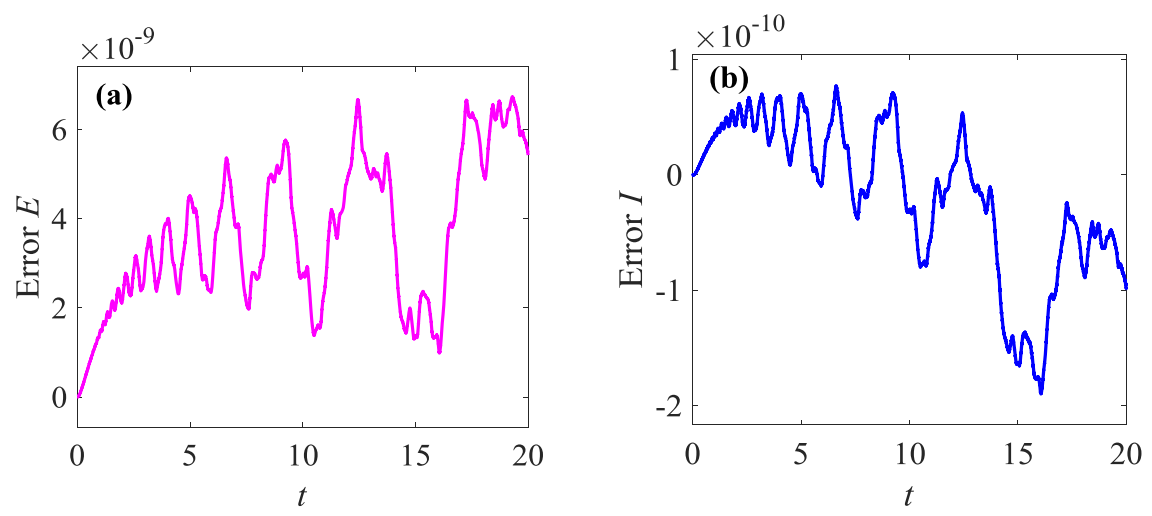

Figure 4. Errors of the discrete global conservation laws with time $0 \leq t \leq 20$ : (a) two-dimensional errors of the discrete global energy conservation law (b) two-dimensional errors of discrete global momentum conservation law.

of the system, which are the global energy conservation law and the global momentum conservation law. Obviously, when we take $10^{-5}$ time steps, the multi-symplectic Fourier pseudo-spectral method to the generalized $\mathrm{KdV}$ equation can preserve the discrete global energy conservation law and global momentum conservation law quite well, which implies long-time numerical stability of the multi-symplectic pseudo-spectral method.

\section{Conclusions}

The generalized KdV-type equation, which can degenerate to the $\mathrm{mKdV}$ equation and the generalized $\mathrm{KdV}$ equation, is given. The variational principle is successfully used to establish a multi-symplectic structure for the $\mathrm{KdV}$-type equation. Based on the variational principle, we also obtain the multi-symplectic structure, local energy conservation law and local momentum conservation law of the generalized KdV-type equation, which are identical to the results by using the HBUCM ${ }^{6,13}$. An explicit multi-symplectic scheme for the generalized KdV equation based on the Fourier pseudo-spectral method and the symplectic Euler scheme are constructed. Through a numerical examination, the explicit multi-symplectic Fourier pseudo-spectral scheme for the generalized KdV equation not only preserve the discrete global energy conservation law and the global momentum conservation law with high accuracy, but show long-time numerical stability as well.

The performance of variational principle is found to be simple and efficient. Moreover, similar to the process of the generalized KdV-type equation, multi-symplectic structures of some NLPDEs can be obtained.

Received: 15 July 2019; Accepted: 15 October 2019;

Published online: 04 November 2019

\section{References}

1. Wadati, M. The modified Korteweg-de Vries equation. Journal of the Physical Society of Japan. 34(5), 1289-1296, https://doi. org/10.1143/JPSJ.34.1289 (1973).

2. Yan, J. L., Zhang, Q., Zhang, Z. Y. \& Liang, D. A new high-order energy-preserving scheme for the modified Korteweg-de Vries equation. Numerical Algorithms 74(3), 659-674, https://doi.org/10.1007/s1107 (2016).

3. Kenig, C. E., Ponce, G. \& Vega, L. Well-posedness and scattering results for the generalized Korteweg-de Vries equation via the contraction principle. Communications on Pure and Applied Mathematics 46(4), 527-620, https://doi.org/10.1002/cpa.3160460405 (1993). 
4. Hu, W. P., Deng, Z. C., Qin, Y. Y. \& Zhang, W. R. Multi-symplectic method for the generalized (2+1)-dimensional KdV-mKdV equation. Acta Mechanica Sinica 28(3), 793-800, https://doi.org/10.1007/s10409-012-0070-2 (2012).

5. Osman, M. S. \& Wazwaz, A. M. An efficient algorithm to construct multi-soliton rational solutions of the $(2+1)$-dimensional KdV equation with variable coefficients. Applied Mathematics and Computation 321, 282-289, https://doi.org/10.1016/j.amc.2017.10.042 (2018).

6. Guo, F. Second order conformal multi-symplectic method for the damped Korteweg-de Vries equation. Chinese Physics B 28(5), 050201, https://doi.org/10.1088/1674-1056/28/5/050201 (2019).

7. Gardner, C. S., Greene, J. M., Kruskal, M. D. \& Miura, R. M. Method for solving the Korteweg-deVries equation. Physical Review Letters 19(19), 1095-1097, https://doi.org/10.1103/PhysRevLett.19.1095 (1967)

8. Wahlquist, H. D. \& Estabrook, F. B. Bäcklund transformation for solutions of the Korteweg-de Vries equation. Physical Review Letters 31, 1386-1390, https://doi.org/10.1103/PhysRevLett.31.1386 (1973).

9. Wang, M. L., Li, X. Z. \& Zhang, J. L. The ( $\left.G^{\prime} / G\right)$-expansion method and traveling wave solutions of nonlinear evolution equations in mathematical physics. Physics Letters A 372, 417-423, https://doi.org/10.1016/j.physleta.2007.07.051 (2008).

10. Hirota, R. Exact solution of the Korteweg-de Vries equation for multiple collisions of solitons. Physics Review Letters 27, 1192-1194, https://doi.org/10.1103/PhysRevLett.27.1192 (1971).

11. Wang, Y. S. \& Hong, J. L. Multi-symplectic algorithms for Hamiltonian partial differential equations. Communication on Applied Mathematics and Computation 27, 163-230, https://doi.org/10.3969/j.issn.1006-6330.2013.02.001 (2013).

12. Dutykh, D., Chhay, M. \& Fedele, F. Geometric numerical schemes for the KdV equation. Computational Mathematics and Mathematical Physics 53(2), 221-236, https://doi.org/10.1134/S0965542513020103 (2013).

13. Yang, X. F., Deng, Z. C., Li, Q. J. \& Wei, Y. Exact solutions and multi-symplectic structure of the generalized KdV-type equation. Advances in Difference Equations, 271, https://doi.org/10.1186/s13662-015-0611-7 (2015)

14. Yang, X. F., Deng, Z. C., Li, Q. J. \& Wei, Y. Exact combined traveling wave solutions and multi-symplectic structure of the variant Boussinesq-Whitham-Broer-Kaup type equations. Communications in Nonlinear Science and Numerical Simulation 36, 1-13, https://doi.org/10.1016/j.cnsns.2015.11.015 (2016).

15. Akram, G. \& Mahak, N. Analytical solution of the Korteweg-de Vries equation and microtubule equation using the first integral method. Optical and Quantum Electronics 50, 145, https://doi.org/10.1007/s11082-018-1401-8 (2018).

16. Abdelsalam, U. M., Allehiany, F. M., Moslem, W. M. \& El-Labany, S. K. Nonlinear structures for extended Korteweg-de Vries equation in multicomponent plasma. Pramana 86(3), 581-597, https://doi.org/10.1007/s12043-015-0990-z (2015).

17. Khan, K., Akbar, M. A. \& Islam, S. M. R. Exact solutions for $(1+1)$-dimensional nonlinear dispersive modified Benjamin-BonaMahony equation and coupled Klein-Gordon equations. SpringerPlus 3(1), 724, https://doi.org/10.1186/2193-1801-3-724 (2014).

18. Wazwaz, A. M. The simplified Hirota's method for studying three extended higher-order KdV-type equations. Journal of Ocean Engineering and Science 1(3), 181-185, https://doi.org/10.1016/j.joes.2016.06.003 (2016).

19. Karakoc, S. B. G. \& Ak, T. Numerical simulation of dispersive shallow water waves with Rosenau-KdV equation. International Journal of Advances in Applied Mathematics and Mechanics 3(3), 32-40, https://doi.org/10.1140/epjp/i2016-16356-3 (2016).

20. Wang, X. F. \& Dai, W. Z. A conservative fourth-order stable finite difference scheme for the generalized Rosenau-KdV equation in both 1D and 2D. Journal of Computational and Applied Mathematics 355, 310-331, https://doi.org/10.1016/j.cam.2019.01.041 (2019).

21. Ascher, U. M. \& McLachlan, R. I. Multisymplectic box schemes and the Korteweg-de Vries equation. Applied Numerical Mathematics 48(3), 255-269, https://doi.org/10.1016/j.apnum.2003.09.002 (2004).

22. Razafindralandy, D., Hamdouni, A. \& Chhay, M. A review of some geometric integrators. Advanced Modeling and Simulation in Engineering Sciences 5(1), 16, https://doi.org/10.1186/s40323-018-0110-y (2018).

23. Song, M., Qian, X., Zhang, H. \& Song, S. Hamiltonian boundary value method for the nonlinear Schrödinger equation and the Korteweg-de Vries equation. Advances in Applied Mathematics and Mechanics 9(4), 868-886, https://doi.org/10.4208/aamm.2015. m1356 (2017).

24. Bridges, T. J. \& Reich, S. Multi-symplectic integrators: numerical schemes for Hamiltonian PDEs that conserve symplecticity. Physics Letters A 284, 184-193, https://doi.org/10.1016/S0375-9601(01)00294-8 (2001).

25. Bridges, T. J. \& Reich, S. Numerical methods for Hamiltonian PDEs. Journal of Physics A: Mathematical and General 39, 5287-5320, https://doi.org/10.1088/0305-4470/39/19/S02 (2006).

26. Reich, S. Multi-symplectic Runge-Kutta collocation methods for Hamiltonian wave equations. Journal of Computational Physics 157, 473-499, https://doi.org/10.1006/jcph.1999.6372 (2000).

27. Moore, B. E. \& Reich, S. Multi-symplectic integration methods for Hamiltonian PDEs. Future Generation Computer Systems 19, 395-402, https://doi.org/10.1016/S0167-739X(02)00166-8 (2003).

28. Chen, J. B. A multi-symplectic pseudospectral method for seismic modeling. Applied Mathematics and Computation 186, 1612-1616, https://doi.org/10.1016/j.amc.2006.08.071 (2007).

29. Lv, Z. Q., Xue, M. \& Wang, Y. S. A new multi-symplectic scheme for the KdV equation. Chinese Physics Letters 28, 060205, https:// doi.org/10.1088/0256-307X/28/6/060205 (2011).

30. Yang, X. F., Deng, Z. C. \& Wei, Y. A Riccati-Bernoulli sub-ODE method for nonlinear partial differential equations and its application. Advances in Difference Equations, 117, https://doi.org/10.1186/s13662-015-0452-4 (2015)

\section{Acknowledgements}

The research is supported by the A Project of Shandong Province Higher Educational Science and Technology Program (J18KB100, J18KA217), the Doctoral Research Foundation of Jining Medical University (2017JYQD22, 2018JYQD03), NSFC cultivation project of Jining Medical University (JYP2018KJ15), Chinese Universities Scientific Fund (2452017373), and the Doctoral Research Foundation of Northwest A\&F University (2452017007).

\section{Author contributions}

Yi Wei analyzed and interpreted the data, and wrote the manuscript; Xing-Qiu Zhang, Zhu-Yan Shao, Jian-Qiang Gao, and Xiao-Feng Yang designed and optimized the algorithm and program. All authors read the manuscript.

\section{Competing interests}

The authors declare no competing interests.

\section{Additional information}

Correspondence and requests for materials should be addressed to Y.W.

Reprints and permissions information is available at www.nature.com/reprints. 
Publisher's note Springer Nature remains neutral with regard to jurisdictional claims in published maps and institutional affiliations.

(c) (i) Open Access This article is licensed under a Creative Commons Attribution 4.0 International License, which permits use, sharing, adaptation, distribution and reproduction in any medium or format, as long as you give appropriate credit to the original author(s) and the source, provide a link to the Creative Commons license, and indicate if changes were made. The images or other third party material in this article are included in the article's Creative Commons license, unless indicated otherwise in a credit line to the material. If material is not included in the article's Creative Commons license and your intended use is not permitted by statutory regulation or exceeds the permitted use, you will need to obtain permission directly from the copyright holder. To view a copy of this license, visit http://creativecommons.org/licenses/by/4.0/.

(c) The Author(s) 2019 